\title{
Distributed Consensus Observer for Multi-Agent Systems With High-Order Integrator Dynamics
}

DOI:

10.1109/TAC.2019.2936555

\section{Document Version}

Accepted author manuscript

Link to publication record in Manchester Research Explorer

\section{Citation for published version (APA):}

Zuo, Z., Defoort, M., Tian, B., \& Ding, Z. (2019). Distributed Consensus Observer for Multi-Agent Systems With High-Order Integrator Dynamics. I E E Transactions on Automatic Control.

https://doi.org/10.1109/TAC.2019.2936555

\section{Published in:}

I E E E Transactions on Automatic Control

\section{Citing this paper}

Please note that where the full-text provided on Manchester Research Explorer is the Author Accepted Manuscript or Proof version this may differ from the final Published version. If citing, it is advised that you check and use the publisher's definitive version.

\section{General rights}

Copyright and moral rights for the publications made accessible in the Research Explorer are retained by the authors and/or other copyright owners and it is a condition of accessing publications that users recognise and abide by the legal requirements associated with these rights.

\section{Takedown policy}

If you believe that this document breaches copyright please refer to the University of Manchester's Takedown Procedures [http://man.ac.uk/04Y6Bo] or contact uml.scholarlycommunications@manchester.ac.uk providing relevant details, so we can investigate your claim.

\section{OPEN ACCESS}




\title{
Distributed Consensus Observer for Multi-Agent Systems With High-Order Integrator Dynamics
}

\author{
Zongyu Zuo, Senior Member, IEEE, Michael Defoort, Bailing Tian, Member, IEEE, and Zhengtao Ding, Senior \\ Member, IEEE
}

\begin{abstract}
This paper presents a distributed consensus observer for multi-agent systems with high-order integrator dynamics to estimate the leader state. Stability analysis is carefully studied to explore the convergence properties under undirected and directed communication, respectively. Using Lyapunov functions, fixed-time (resp. finite-time) stability is guaranteed for the undirected (resp. directed) interaction topology. Finally, simulation results are presented to demonstrate the theoretical findings.
\end{abstract}

Index Terms-Finite time, Fixed time, High-order system, Lyapunov function, Multi-agent system, Observer, Stability

\section{INTRODUCTION}

Cooperative control of multi-agent systems has received considerable attention in the recent decade. In the modern context of multi-agent systems, numerous results [1], [2], [3] have been obtained for the consensus problem which aims to steer the states or outputs of all agents subject to certain communication topology to the quantities of interest. Note that in real systems, it is often inhibitive to have the measurements of all system states for consensus control, such as the formation flying control of UAVs whose relative positions are available for control [4]. In such a case, relative state feedback protocol clearly are unapplicable, but the observer-based or output feedback control idea can provide a viable solution. In [5], [6], observer-based protocols were developed for leader-follower consensus problem of multi-agent systems in the presence of Lipschitz nonlinearity and the asymptotic stability is achieved by output feedback. In [7], finitetime observers were used to reconstruct the states of the agents for consensus design. During recent years, the fixed-time stability [8], as an extension of the finite-time stability, has been defined, assuming that the global finite settling time is uniformly bounded by a constant without dependence on initial conditions. Moreover, the corresponding Lyapunov criteria [8], [9], [10] have been developed. Although many fixed-time leader-follower consensus [11], [12], [13], [14], [15], [16], based on local state information, have been reported, few efforts are deployed on the fixed-time consensus by output feedback, i.e., local output information. But it might be expected that the fixed-time observers or differentiators, such as [17], [18], could provide a possibly feasible solution to the fixed-time output feedback consensus problem.

This paper focuses on a distributed consensus observer, which is implemented in a group of follower agents to reconstruct the leader state. Indeed, in the leader-follower consensus problem, a common assumption is that only a portion of followers can get access to the leader's information. However, if there are measuring biases

This work was supported by the National Natural Science Foundation of China (No.61673034).

Z. Zuo is with the Seventh Research Division, Beihang University (BUAA), Beijing 100191, China. (zzybobby@buaa.edu.cn)

M. Defoort is with LAMIH, CNRS UMR 8201, Polytechnic University of Hauts-de-France, Le Mont Houy, 59313 Valenciennes Cedex 9, France. (michael.defoort@uphf.fr)

B. Tian is with School of Electric and Automation Engineering, Tianjin University, Tianjin 300072, China. (bailing_tian@tju.edu.cn)

Z. Ding is with School of Electrical and Electronic Engineering, University of Manchester, Sackville Street Building, Manchester M13 9PL, UK. (zhengtao.ding@manchester.ac.uk) among agents, some misbehaviors like a distorted final shape and a steady-state motion of the group formation may occur [19]. An alternative way of overcoming this issue is to use the consensus observer to estimate the leader information for each agent in a distributed fashion and use the estimated information for consensus control and the like. The terminology consensus observer is adopted in this paper to distinguish the conventional concept of state observer which is used to reconstruct the unmeasured states of a given system. It has been shown in [20], [21], [22] that the communication loop problem, encountered in [13], [23], [24], can be technically eluded with the introduction of a consensus observer. In the works [20], [21], first- and second-order fixed-time observers have been proposed in a distributed manner for the fixed-time leader-follower consensus design. It must be noted that the observer proposed by $\mathrm{Fu}$ and Wang [21] is a standard fixed-time stabilization structure which generally consists of two feedback terms: one with fractional exponent and the other with exponent greater than 1 , whereas the observer proposed in this paper takes just one power exponent and therefore is simpler in design and parameter tuning. This also implies that the stability analysis in [21] is not applicable for our case. The difficulties arise from the noncanonical structure studied in this paper for the fixed-time stabilization design. Note also that the consensus observer proposed in our recent work [22], which can be viewed as a special case of the one developed in this paper, achieves fixed-time stability by assuming that the interaction topology among followers is undirected and connected. Unfortunately, the proof, based on symmetric topology, is not applicable for the directed one, since the information flow in digraph is unidirectional and the communication topology is asymmetric. It has been shown in the existing literature [25] that the stability proof for multi-agent systems subject to directed topology is nontrivial, especially the fixed-stability is much more involved.

The contribution of this paper mainly deals with the distributed consensus observer design which is used to reconstruct the leader information in the leader-follower context. Within the Lyapunov framework, the stability of the observation errors is carefully analyzed under undirected and directed interaction topology, respectively. The fixed-time convergence of the estimation errors to zero can be guaranteed under undirected and connected information flow. More interestingly and importantly, it is found that under directed topology containing a spanning tree, the consensus observer can achieve the fixed-time set-attractivity and asymptotic stability at the origin or the finite-time stability at the origin by just tuning an exponent parameter. A set of simulation results validate the theoretical findings.

\section{Preliminaries and Problem Statement}

Notation: For any non-negative real number $\alpha$, the function $x \mapsto\lceil x\rfloor^{\alpha}$ is defined as $\lceil x\rfloor^{\alpha}=|x|^{\alpha} \operatorname{sign}(x)$ for any $x \in$ $\mathbb{R}$. In terms of the definition, we have $x\lceil x\rfloor^{\alpha}=|x|^{\alpha+1}$. For any $x=\left[x_{1}, x_{2}, \ldots, x_{N}\right]^{T} \in \mathbb{R}^{N}$, we define $\lceil x\rfloor^{\alpha}=$ $\left[\operatorname{sign}\left(x_{1}\right)\left|x_{1}\right|^{\alpha}, \operatorname{sign}\left(x_{2}\right)\left|x_{2}\right|^{\alpha}, \ldots, \operatorname{sign}\left(x_{N}\right)\left|x_{N}\right|^{\alpha}\right]^{T}$. For $p>0$, $\|x\|_{p}$ denotes the $p$-norm of vector $x$. For matrix $A \in \mathbb{R}^{n \times n}, \lambda_{\max }^{A}$ and $\lambda_{\max }^{A}$ denote the maximum and the minimum eigenvalue of $A$, respectively. Furthermore, $\|A\|_{p}$ represents the induced $p$-norm 
of matrix $A$. Throughout the paper, 1 denotes the vector with all elements one.

\section{A. Definitions and Lemmas}

Consider the system of differential equations

$$
\dot{x}(t)=f(t, x), \quad x(0)=x_{0},
$$

where $x \in \mathbb{R}^{n}$ and $f: \mathbb{R}_{+} \times \mathbb{R}^{n} \rightarrow \mathbb{R}^{n}$ is a nonlinear function. The solutions of (1) are understood in the sense of Filippov [26] if $f(t, x)$ is discontinuous. Suppose the origin is an equilibrium point of (1).

Several definitions and lemmas are invoked from the existing literature.

Definition 1: [8] The origin of system (1) is said to be globally finite-time stable if it is globally asymptotically stable and any solution $x\left(t, x_{0}\right)$ of (1) reaches the origin at some finite moment, i.e., $x\left(t, x_{0}\right)=0, t \geq T\left(x_{0}\right)$, where $T: \mathbb{R}^{n} \rightarrow \mathbb{R}_{+} \cup 0$ is the settling time function.

Definition 2: [8] The origin of system (1) is said to be globally fixedtime stable if it is globally uniformly finite-time stable and the settling time function $T\left(x_{0}\right)$ is globally bounded, i.e., there exists a finite constant $T_{\max } \in \mathbb{R}_{+}$such that $T \leq T_{\max }$ and $x(t)=0$ for all $t \geq T$ and $x_{0} \in \mathbb{R}^{n}$.

Definition 3: [8] The set $\mathcal{S}$ is said to be globally finite-time attractive for system (1) if any solution $x\left(t, x_{0}\right)$ of (1) reaches $\mathcal{S}$ in some finite time moment $T\left(x_{0}\right)$ and remains there for $t \geq T\left(x_{0}\right), T: \mathbb{R}^{n} \rightarrow$ $\mathbb{R}_{+} \cup 0$ is the settling time function.

Definition 4: [8] The set $\mathcal{S}$ is said to be fixed-time attractive for system (1) if it is globally finite-time attractive and the settling-time function $T\left(x_{0}\right)$ is globally bounded by some constant $T_{\max }>0$.

Lemma 1: [27] Suppose there exists a continuous positive definite function $V(x): \mathbb{R}^{n} \rightarrow \mathbb{R}$ such that there exist real number $c>0$ and $\alpha \in(0,1)$ such that $\dot{V}(x)+c(V(x))^{\alpha} \leq 0, x \in \mathbb{R}^{n} \backslash\{0\}$. Then the origin is a globally finite-time stable equilibrium of (1) and the settling time is $T\left(x_{0}\right) \leq \frac{1}{c(1-\alpha)} V\left(x_{0}\right)^{(1-\alpha)}$.

Lemma 2: [9] If there exists a continuous radially unbounded and positive definite function $V(x)$ such that $\dot{V}(x) \leq-\alpha V^{p}-\beta V^{q}$ for some $\alpha, \beta>0, p>1,0<q<1$, then the origin of this system (1) is globally fixed-time stable and the settling time function $T$ can be estimated by $T \leq \frac{1}{\alpha(p-1)}+\frac{1}{\beta(1-q)}$. In particular, if the constants $p$ and $q$ are of the form $p=1+\frac{1}{2 \varrho}$ and $q=1-\frac{1}{2 \varrho}, \varrho>1$, a more exact estimate of the settling time can be given as $T \leq \frac{\pi \varrho}{\sqrt{\alpha \beta}}$.

The following three lemmas will be useful in the stability analysis.

Lemma 3: [13] Let $\xi_{1}, \xi_{2}, \ldots, \xi_{N} \geq 0$ and $0<p \leq 1$. Then, $\sum_{i=1}^{N} \xi_{i}^{p} \geq\left(\sum_{i=1}^{N} \xi_{i}\right)^{p}$.

Lemma 4: [21] Let $\xi_{1}, \xi_{2}, \ldots, \xi_{N} \geq 0$ and $p>1$. Then, $N^{1-p}\left(\sum_{i=1}^{N} \xi_{i}\right)^{p} \leq \sum_{i=1}^{N} \xi_{i}^{p} \leq\left(\sum_{i=1}^{N} \xi_{i}\right)^{p}$.

Lemma 5: [28] Let $c$ and $d$ be positive constants. Given any real number $\zeta>0$ and for $\forall x, y \in \mathbb{R}$, the inequality $|x|^{c}|y|^{d} \leq$ $\frac{c}{c+d} \zeta|x|^{c+d}+\frac{d}{c+d} \zeta^{-1}|y|^{c+d}$ holds.

\section{B. Interaction Topology}

Consider $N+1$ agents with one leader and $N$ followers labeled by 0 and $i \in \mathcal{V}=\{1,2, \ldots, N\}$, respectively. The communication connection between the followers can be described by an edge set $\mathcal{E} \subseteq \mathcal{V} \times \mathcal{V}$ of a digraph $\mathcal{G}=\{\mathcal{V}, \mathcal{E}\}$. The weighted adjacency matrix $A=\left[a_{i j}\right] \in \mathbb{R}^{N \times N}$ is defined by $a_{i j}>0$ if the agent $i$ can receive information from the agent $j$, otherwise $a_{i j}=0$. For an undirected graph as a special case, $a_{i j}=a_{j i}$ holds. Here, it is assumed that $a_{i i}=0$ (i.e., self loops are not allowed). A directed path from node $v_{1}$ to $v_{l}$ is a sequence of ordered edges $\left(v_{k}, v_{k+1}\right), k=$ $1,2, \ldots, l-1$, where $v_{k} \in \mathcal{V}$. A directed graph contains a directed spanning tree if there exists at least one node that has a directed path to any other node. Denote by $D=\operatorname{diag}\left\{d_{1}, d_{2}, \ldots, d_{N}\right\}$ the degree diagonal matrix, where $d_{i}=\sum_{j=1}^{N} a_{i j}$ for $i=1,2, \ldots, N$. The graph Laplacian matrix is $L=\left[l_{i j}\right]=D-A$ with appropriate dimension, i.e., $l_{i j}=-a_{i j}$ for $i \neq j$ and $l_{i i}=\sum_{j=1}^{N} a_{i j}$. Let the diagonal matrix $B=\operatorname{diag}\left\{b_{1}, b_{2}, \ldots, b_{N}\right\}$ be the interconnection relationship between the leader and followers, where $b_{i}>0$ if the information of the leader is accessible by the $i$ th follower, otherwise $b_{i}=0$. Denote by $\mathcal{G}^{e}$ the extended digraph incorporating the leader into $\mathcal{G}$ by adding a link $\left(v_{0}, v_{i}\right)$ whenever $b_{i}>0$.

\section{Problem Formulation}

The dynamics of the leader is described as follows

$$
\dot{x}_{0,1}(t)=x_{0,2}(t), \dot{x}_{0,2}(t)=x_{0,3}(t), \cdots, \dot{x}_{0, n}(t)=u_{0}(t),
$$

where $x_{0}=\left[x_{0,1}, x_{0,2}, \ldots, x_{0, n}\right]^{T} \in \mathbb{R}^{n}$ is the state vector and $u_{0} \in \mathbb{R}$ is the control input, respectively, of the leader.

Assumption 1: The input $u_{0}$ of the leader is unknown to any followers but its upper bound, denoted by $\bar{u}_{0}$, can be accessible by the $i$ th follower if $b_{i} \neq 0, i \in \mathcal{V}$.

In consideration that the leader information is available not to all followers but to only a portion of them, a consensus observer is required by each follower to estimate the leader state in a distributed manner.

\section{Distributed CONSEnsus ObSERVER}

Denote by $\hat{x}_{0, k}^{i}$ the estimate of the leader's state $x_{0, k}, k=1,2, \ldots, n$ for the $i$ th follower, $i \in \mathcal{V}$. A distributed fixed-time observer takes the following structure:

$$
\begin{aligned}
\dot{\hat{x}}_{0, k}^{i}= & \hat{x}_{0, k+1}^{i} \\
& +\alpha_{k} \operatorname{sign}\left(\sum_{j=1}^{N} a_{i j}\left(\hat{x}_{0, k}^{j}-\hat{x}_{0, k}^{i}\right)+b_{i}\left(x_{0, k}-\hat{x}_{0, k}^{i}\right)\right) \\
& +\beta_{k}\left[\sum_{j=1}^{N} a_{i j}\left(\hat{x}_{0, k}^{j}-\hat{x}_{0, k}^{i}\right)+b_{i}\left(x_{0, k}-\hat{x}_{0, k}^{i}\right)\right]^{\gamma}, \\
\dot{\hat{x}}_{0, n}^{i}= & \alpha_{n} \operatorname{sign}\left(\sum_{j=1}^{N} a_{i j}\left(\hat{x}_{0, n}^{j}-\hat{x}_{0, n}^{i}\right)+b_{i}\left(x_{0, n}-\hat{x}_{0, n}^{i}\right)\right) \\
& +\beta_{n}\left[\sum_{j=1}^{N} a_{i j}\left(\hat{x}_{0, n}^{j}-\hat{x}_{0, n}^{i}\right)+b_{i}\left(x_{0, n}-\hat{x}_{0, n}^{i}\right)\right]^{\gamma} .
\end{aligned}
$$

Let the observation errors be

$$
\tilde{x}_{0, k}^{i}=\hat{x}_{0, k}^{i}-x_{0, k} .
$$

With (4), the observation error dynamics can be derived as

$$
\begin{aligned}
\dot{\tilde{x}}_{0, k}^{i}= & \tilde{x}_{0, k+1}^{i}+\alpha_{k} \operatorname{sign}\left(\sum_{j=1}^{N} a_{i j}\left(\tilde{x}_{0, k}^{j}-\tilde{x}_{0, k}^{i}\right)-b_{i} \tilde{x}_{0, k}^{i}\right) \\
& +\beta_{k}\left[\sum_{j=1}^{N} a_{i j}\left(\tilde{x}_{0, k}^{j}-\tilde{x}_{0, k}^{i}\right)-b_{i} \tilde{x}_{0, k}^{i}\right]^{\gamma}, \\
\dot{\tilde{x}}_{0, n}^{i}= & \alpha_{n} \operatorname{sign}(k=1,2, \ldots, n-1), \\
& \left.+\beta_{j=1}^{N} a_{i j}\left(\sum_{j=1}^{N} a_{i j}^{j}\left(\tilde{x}_{0, n}^{j}-\tilde{x}_{0, n}^{i}\right)-b_{i} \tilde{x}_{0, n}^{i}\right)-b_{i} \tilde{x}_{0, n}^{i}\right]^{\gamma}-u_{0} .
\end{aligned}
$$


Let $\tilde{\chi}_{i} \triangleq\left[\tilde{x}_{0, i}^{1}, \tilde{x}_{0, i}^{2}, \ldots, \tilde{x}_{0, i}^{N}\right]^{T}$ for $i=1,2, \ldots, n$ be the observation error vectors, and a compact form can be derived as

$$
\begin{gathered}
\dot{\tilde{\chi}}_{k}=\tilde{\chi}_{k+1}-\alpha_{k} \operatorname{sign}\left(H \tilde{\chi}_{k}\right)-\beta_{k}\left\lceil H \tilde{\chi}_{k}\right\rfloor^{\gamma}, \\
(k=1,2, \ldots, n-1), \\
\dot{\tilde{\chi}}_{n}=-\alpha_{n} \operatorname{sign}\left(H \tilde{\chi}_{n}\right)-\beta_{n}\left\lceil H \tilde{\chi}_{n}\right\rfloor^{\gamma}-\mathbf{1} u_{0} .
\end{gathered}
$$

with $H \triangleq L+B$.

Let the observation error disagreement vector be $z_{l} \triangleq$ $\left[z_{l}^{1}, z_{l}^{2}, \ldots, z_{l}^{N}\right]^{T}$ with its element defined by

$$
z_{l}^{i} \triangleq \sum_{j=1}^{N} a_{i j}\left(\tilde{x}_{0, l}^{j}-\tilde{x}_{0, l}^{i}\right)-b_{i} \tilde{x}_{0, l}^{i}
$$

where $l=1,2, \ldots, n$ and $i \in \mathcal{V}$.

Remark 1: Without ambiguity, we use the terminology disagreement here although $z_{l}^{i}$ in (7) receives the leader's information, since the goal of each consensus observer is to achieve the same value with the leader. In addition, the definition of observation error disagreement (7) helps in simplifying the derivation of disagreement dynamics and the stability analysis.

Then, the disagreement dynamics can be computed as

$$
\begin{aligned}
\dot{z}_{k}^{i}= & \sum_{j=1}^{N} a_{i j}\left(\dot{\tilde{x}}_{0, k}^{j}-\dot{\tilde{x}}_{0, k}^{i}\right)-b_{i} \dot{\tilde{x}}_{0, k}^{i} \\
= & z_{k+1}^{i} \\
& +\alpha_{k}\left(\sum_{j=1}^{N} a_{i j}\left(\operatorname{sign}\left(z_{k}^{j}\right)-\operatorname{sign}\left(z_{k}^{i}\right)\right)-b_{i} \operatorname{sign}\left(z_{k}^{i}\right)\right) \\
& +\beta_{k}\left(\sum_{j=1}^{N} a_{i j}\left(\left\lceil z_{k}^{j}\right\rfloor^{\gamma}-\left\lceil z_{k}^{i}\right\rfloor^{\gamma}-b_{i}\left\lceil z_{k}^{i}\right\rfloor^{\gamma}\right)\right. \\
& (k=1,2, \ldots, n-1) \\
\dot{z}_{n}^{i}= & \alpha_{n}\left(\sum_{j=1}^{N} a_{i j}\left(\operatorname{sign}\left(z_{n}^{j}\right)-\operatorname{sign}\left(z_{n}^{i}\right)\right)-b_{i} \operatorname{sign}\left(z_{n}^{i}\right)\right) \\
& +\beta_{n}\left(\sum_{j=1}^{N} a_{i j}\left(\left\lceil z_{n}^{j}\right\rfloor^{\gamma}-\left\lceil z_{n}^{i}\right\rfloor^{\gamma}-b_{i}\left\lceil z_{n}^{i}\right\rfloor^{\gamma}\right)\right. \\
& -b_{n} u_{0}
\end{aligned}
$$

Remark 2: Obviously, $\tilde{\chi}_{i}=0$ implies $\hat{x}_{0, k}^{i}=x_{0, k}$, i.e., the consensus observer achieves the accurate estimation of the leader state. It will be shown in the next section that the fixed-time convergence of (6) under undirected graph can be derived. However, for the directed graph, the establishment of $\tilde{\chi}_{i}=0$ is much more involved and the stability proof is nontrivial. To solve this problem, the disagreement dynamics (8) will be employed for stability analysis. Observing from (7) that $z_{i}=-H \tilde{\chi}_{i}$, the convergence of $\tilde{\chi}_{i}$ follows from the convergence of $z_{i}$ provided that certain conditions are satisfied.

Remark 3: It is worth mentioning that observer (3) is distributed. Otherwise, if $x_{0, k}$ is transmitted from follower $i$ to follower $j$, instead of $\hat{x}_{0, k}^{i}$, the scheme becomes a centralized one. Here, the proposed scheme combines information both from the leader (if there is a direct access) and information from the neighboring followers. This may be useful in the case of faults (for instance a communication link disappears or becomes intermittent) since it may add some redundancy of information. Furthermore, it is of some interest to have the same algorithm for each agent without distinguishing agents with direct communication with the leader. It may increase security since the leader is not bringing in light.

\section{StabiLity ANALYSis}

In this section, a rigorous stability analysis is performed to show some novel features of the proposed consensus observer (3) with respect to different interaction graphs.

\section{A. Undirected Interaction Topology}

If graph $\mathcal{G}$ is undirected, the following assumption is needed to formulate our first result.

Assumption 2: Graph $\mathcal{G}$ is connected and at least one follower in graph $\mathcal{G}$ can get access to the leader state, i.e., $B \neq 0$.

Remark 4: Assumption 2 is necessary for the design objective of the consensus observer. Otherwise, the behavior of followers is involved independently of the leader.

Lemma 6: [29] If Assumption 2 holds, then $H=L+B>0$.

For the undirected graph, the fixed-time stability of (6) with $\gamma=2$ has been reported in the work [22]. Here, we will generalize the result in [22].

Theorem 1: If Assumptions 1 and 2 hold and the observer gains satisfy

$$
\begin{aligned}
\beta_{k} & =\frac{\varepsilon N^{\frac{1-\gamma}{2}}}{\left(2 \lambda_{\min }^{H}\right)^{\frac{1+\gamma}{2}}}, \quad \forall k=1,2, \ldots, n, \\
\alpha_{k} & =\varepsilon \sqrt{\frac{\lambda_{\max }^{H}}{2 \lambda_{\min }^{H}}}, \quad \forall k=1,2, \ldots, n-1, \\
\alpha_{n} & =\bar{u}_{0}+\varepsilon \sqrt{\frac{\lambda_{\max }^{H}}{2 \lambda_{\min }^{H}}}, \quad \gamma>1,
\end{aligned}
$$

with $\varepsilon>0$, then the distributed observer (3) achieves the fixed-time stability of the observation errors at the origin with the settling time bounded by

$$
T_{o}:=\frac{n}{\varepsilon(\gamma-1)} .
$$

Proof: Consider the following Lyapunov function candidate $V_{n}=\frac{1}{2} \tilde{\chi}_{n}^{T} H \tilde{\chi}_{n}$ and its time derivative is

$$
\begin{aligned}
\dot{V}_{n} & =\tilde{\chi}_{n}^{T} H\left(-\alpha_{n} \operatorname{sign}\left(H \tilde{\chi}_{n}\right)-\beta_{n}\left\lceil H \tilde{\chi}_{n}\right\rfloor^{\gamma}\right)-\tilde{\chi}_{n}^{T} H \mathbf{1} u_{0} \\
& \leq-\alpha_{n}\left\|H \tilde{\chi}_{n}\right\|_{1}-\beta_{n}\left\|H \tilde{\chi}_{n}\right\|_{1+\gamma}^{1+\gamma}+\bar{u}_{0}\left\|H \tilde{\chi}_{n}\right\|_{1} \\
& \leq-\left(\alpha_{n}-\bar{u}_{0}\right)\left\|H \tilde{\chi}_{n}\right\|_{1}-\beta_{n} N^{\frac{1-\gamma}{2}}\left\|H \tilde{\chi}_{n}\right\|_{2}^{1+\gamma} \\
& \leq-\left(\alpha_{n}-\bar{u}_{0}\right)\left\|H \tilde{\chi}_{n}\right\|_{1}-\beta_{n} N^{\frac{1-\gamma}{2}}\left(2 \lambda_{\min }^{H}\right)^{\frac{1+\gamma}{2}} V_{n}^{\frac{1+\gamma}{2}} \\
& \leq-\varepsilon V_{n}^{\frac{1}{2}}-\varepsilon V_{n}^{\frac{1+\gamma}{2}}
\end{aligned}
$$

where $\left\|\tilde{\chi}_{n}\right\|_{2}^{1+\gamma} \leq N^{(\gamma-1) / 2}\left\|\tilde{\chi}_{n}\right\|_{1+\gamma}^{1+\gamma}$ and $\left\|H \tilde{\chi}_{n}\right\|_{2} \leq \sqrt{2 \lambda_{\min }^{H} V_{n}}$ are used. Hence, Lemma 2 guarantees that $\tilde{\chi}_{n}$ is fixed-time stable at the origin with the settling time bounded by $T_{1}=\frac{1}{\varepsilon(\gamma-1)}$.

After the convergence of $\tilde{\chi}_{n}$, the dynamics of $\tilde{\chi}_{n-1}$ reduces to

$$
\dot{\tilde{\chi}}_{n-1}=-\alpha_{n-1} \operatorname{sign}\left(H \tilde{\chi}_{n-1}\right)-\beta_{n-1}\left\lceil H \tilde{\chi}_{n-1}\right\rfloor^{\gamma} .
$$

Similar, we have that $\tilde{\chi}_{n-1}$ converges to zero in a fixed-time bounded by $T_{2}=2 T_{1}$. Recursively, we have that $\tilde{\chi}_{1}$ converges to zero within a fixed time horizon bounded by $T_{o}:=T_{n}=n T_{1}$. This completes the proof.

Remark 5: Note that conditions (10)-(11) proposed in Theorem 1 are a bit strong in the sense that global information like $N, \lambda_{\max }^{H}$ and $\lambda_{\text {min }}^{H}$ are required to guarantee the fixed-time convergence. Instead, if $\alpha_{k}=0, k=1,2, \ldots, n-1, \alpha_{n}=\bar{u}_{0}, \beta_{k}>0, k=1,2, \ldots, n$ and $\gamma=1$, then consensus observer (3) becomes a linear one and the asymptotic stability is ensured. However, Theorem 1 provides an explicit estimation of the settling time that can be prescribed by tuning $\varepsilon$ and $\gamma$ according to (12). 


\section{B. Directed Interaction Topology}

If graph $\mathcal{G}$ is directed, the resultant stability analysis is nontrivial. To streamline the analysis, a reasonable assumption is invoked.

Assumption 3: Graph $\mathcal{G}^{e}$ contains a spanning tree with the leader as the root node, i.e., $B \neq 0$.

Lemma 7: [25] If Assumption 3 holds, then $H=L+B$ is of full rank. Furthermore, define

$$
\begin{aligned}
{\left[p_{1}, p_{2}, \ldots, p_{N}\right]^{T} } & =H^{-T} \mathbf{1}_{N}, \\
P & =\operatorname{diag}\left\{p_{1}, p_{2}, \ldots, p_{N}\right\}, \\
Q & =\frac{P H+H^{T} P}{2} .
\end{aligned}
$$

Then, the diagonal matrix $P$ and symmetric matrix $Q$ are both positive definite.

Before presenting the main results of this paper, an induction analysis for $\gamma>1$ is presented for the convenience of presentation.

Step 1 We may try the Lyapunov function candidate

$$
V_{n}=\sum_{i=1}^{N} p_{i}\left(\alpha_{n}\left|z_{n}^{i}\right|+\frac{\beta_{n}}{1+\gamma}\left|z_{n}^{i}\right|^{1+\gamma}\right)
$$

Then, we have

$$
\begin{aligned}
\dot{V}_{n}= & \sum_{i=1}^{N} p_{i}\left(\alpha_{n} \operatorname{sign}\left(z_{n}^{i}\right)+\beta_{n}\left\lceil z_{n}^{i}\right\rfloor^{\gamma}\right) \times \\
& \left(\alpha_{n}\left(\sum_{j=1}^{N} a_{i j}\left(\operatorname{sign}\left(z_{n}^{j}\right)-\operatorname{sign}\left(z_{n}^{i}\right)\right)-b_{i} \operatorname{sign}\left(z_{n}^{i}\right)\right)\right. \\
& \left.+\beta_{n}\left(\sum_{j=1}^{N} a_{i j}\left(\left\lceil z_{n}^{j}\right\rfloor^{\gamma}-\left\lceil z_{n}^{i}\right\rfloor^{\gamma}\right)-b_{i}\left\lceil z_{n}^{j}\right\rfloor^{\gamma}\right)-b_{n} u_{0}\right) \\
= & -\left(\alpha_{n} \operatorname{sign}\left(z_{n}\right)+\beta_{n}\left\lceil z_{n}\right\rfloor^{\gamma}\right)^{T} P H\left(\alpha_{n} \operatorname{sign}\left(z_{n}\right)+\beta_{n}\left\lceil z_{n}\right\rfloor^{\gamma}\right) \\
& -\sum_{i=1}^{N} p_{i}\left(\alpha_{n} \operatorname{sign}\left(z_{n}^{i}\right)+\beta_{n}\left\lceil z_{n}^{i}\right\rfloor^{\gamma}\right) b_{n} u_{0} \\
= & -\left(\alpha_{n} \operatorname{sign}\left(z_{n}\right)+\beta_{n}\left\lceil z_{n}\right\rfloor^{\gamma}\right)^{T} Q\left(\alpha_{n} \operatorname{sign}\left(z_{n}\right)+\beta_{n}\left\lceil z_{n}\right\rfloor^{\gamma}\right) \\
& -\sum_{i=1}^{N} p_{i}\left(\alpha_{n} \operatorname{sign}\left(z_{n}^{i}\right)+\beta_{n}\left\lceil z_{n}^{i}\right\rfloor^{\gamma}\right) b_{n} u_{0} \\
\leq & -\lambda_{\min }^{Q} \sum_{i=1}^{N}\left(\alpha_{n}^{2}+2 \alpha_{n} \beta_{n}\left|z_{n}^{i}\right|^{\gamma}+\beta_{n}^{2}\left|z_{n}^{i}\right|^{2 \gamma}\right) \\
& +\alpha_{n} b_{n} \bar{u}_{0} N \bar{p}+\beta_{n} b_{n} \bar{u}_{0} \bar{p} \sum_{i=1}^{N}\left|z_{n}^{i}\right|^{\gamma} \\
&
\end{aligned}
$$

where $\bar{p} \triangleq \max \left\{p_{1}, p_{2}, \ldots, p_{N}\right\}$ and $Q=\left(P H+H^{T} P\right) / 2$ is invoked. Let $\alpha_{n} \triangleq b_{n} \bar{u}_{0} \bar{p} / \lambda_{\min }^{Q}$, and for $\gamma>1, \dot{V}_{n}$ verifies

$$
\begin{aligned}
\dot{V}_{n} \leq & -\lambda_{\min }^{Q} \beta_{n} \sum_{i=1}^{N}\left(\alpha_{n}\left|z_{n}^{i}\right|^{\gamma}+\beta_{n}\left|z_{n}^{i}\right|^{2 \gamma}\right) \\
\leq & -\lambda_{\min }^{Q} \alpha_{n} \beta_{n} N^{1-\gamma}\left(\sum_{i=1}^{N}\left|z_{n}^{i}\right|\right)^{\gamma} \\
& -\lambda_{\min }^{Q} \beta_{n}^{2} N^{1-2 \gamma}\left(\sum_{i=1}^{N}\left|z_{n}^{i}\right|\right)^{2 \gamma}
\end{aligned}
$$

where Lemma 4 has been inserted to obtain the second inequality. Case i) For $\sum_{i=1}^{N}\left|z_{n}^{i}\right|>1$, from (15) we have

$$
\begin{aligned}
V_{n} & \leq \alpha_{n} \bar{p} \sum_{i=1}^{N}\left|z_{n}^{i}\right|+\frac{\beta_{n} \bar{p}}{1+\gamma}\left(\sum_{i=1}^{N}\left|z_{n}^{i}\right|\right)^{1+\gamma} \\
& \leq \bar{p}\left(\alpha_{n}+\frac{\beta_{n}}{1+\gamma}\right)\left(\sum_{i=1}^{N}\left|z_{n}^{i}\right|\right)^{1+\gamma}
\end{aligned}
$$

Then, we have

$$
\sum_{i=1}^{N}\left|z_{n}^{i}\right| \geq\left(\frac{V_{n}}{\bar{p}\left(\alpha_{n}+\frac{\beta_{n}}{1+\gamma}\right)}\right)^{\frac{1}{1+\gamma}}
$$

Substituting (17) into (16) yields

$$
\begin{aligned}
\dot{V}_{n} \leq & -\frac{\lambda_{\min }^{Q} \alpha_{n} \beta_{n} N^{1-\gamma}}{\bar{p}^{\frac{1}{1+\gamma}}\left(\alpha_{n}+\frac{\beta_{n}}{1+\gamma}\right)^{\frac{1}{1+\gamma}}} V_{n}^{\frac{\gamma}{1+\gamma}} \\
& -\frac{\lambda_{\min }^{Q} \beta_{n}^{2} N^{1-2 \gamma}}{\bar{p}^{\frac{1}{1+\gamma}}\left(\alpha_{n}+\frac{\beta_{n}}{1+\gamma}\right)^{\frac{1}{1+\gamma}}} V_{n}^{\frac{2 \gamma}{1+\gamma}}
\end{aligned}
$$

Case ii) For $\sum_{i=1}^{N}\left|z_{n}^{i}\right| \leq 1$, from (15) we have

$$
V_{n} \leq \bar{p}\left(\alpha_{n}+\frac{\beta_{n}}{1+\gamma}\right) \sum_{i=1}^{N}\left|z_{n}^{i}\right|
$$

and

$$
\sum_{i=1}^{N}\left|z_{n}^{i}\right| \geq\left(\frac{V_{n}}{\bar{p}\left(\alpha_{n}+\frac{\beta_{n}}{1+\gamma}\right)}\right)
$$

Substituting (19) into (16) obtains

$$
\dot{V}_{n} \leq-\frac{\lambda_{\min }^{Q} \alpha_{n} \beta_{n} N^{1-\gamma}}{\bar{p}\left(\alpha_{n}+\frac{\beta_{n}}{1+\gamma}\right)} V_{n}^{\gamma}-\frac{\lambda_{\min }^{Q} \beta_{n}^{2} N^{1-2 \gamma}}{\bar{p}\left(\alpha_{n}+\frac{\beta_{n}}{1+\gamma}\right)} V_{n}^{2 \gamma}
$$

Step $k$ Consider the following Lyapunov function candidate

$$
V_{k}=\sum_{i=1}^{N} p_{i}\left(\alpha_{k}\left|z_{k}^{i}\right|+\frac{\beta_{k}}{1+\gamma}\left|z_{k}^{i}\right|^{1+\gamma}\right)
$$

where $k=1,2, \ldots, N-1$. Differentiating (15) along (8) obtains

$$
\begin{aligned}
\dot{V}_{k}= & \sum_{i=1}^{N} p_{i}\left(\alpha_{k} \operatorname{sign}\left(z_{k}^{i}\right)+\beta_{k}\left\lceil z_{k}^{i}\right\rfloor^{\gamma}\right)\left(z_{k+1}^{i}\right. \\
& +\alpha_{k}\left(\sum_{j=1}^{N} a_{i j}\left(\operatorname{sign}\left(z_{k}^{j}\right)-\operatorname{sign}\left(z_{k}^{i}\right)\right)-b_{k} \operatorname{sign}\left(z_{k}^{i}\right)\right) \\
& \left.+\beta_{k}\left(\sum_{j=1}^{N} a_{i j}\left(\left\lceil z_{k}^{j}\right\rfloor^{\gamma}-\left\lceil z_{k}^{i}\right\rfloor^{\gamma}\right)-b_{k}\left\lceil z_{k}^{j}\right\rfloor^{\gamma}\right)\right) \\
= & -\left(\alpha_{k} \operatorname{sign}\left(z_{k}\right)+\beta_{k}\left\lceil z_{k}\right\rfloor^{\gamma}\right)^{T} Q\left(\alpha_{k} \operatorname{sign}\left(z_{k}\right)+\beta_{k}\left\lceil z_{k}\right\rfloor^{\gamma}\right) \\
& +\sum_{i=1}^{N} p_{i}\left(\alpha_{k} \operatorname{sign}\left(z_{k}^{i}\right)+\beta_{k}\left\lceil z_{k}^{i}\right\rfloor^{\gamma}\right) z_{k+1}^{i} \\
\leq & -\lambda_{\min }^{Q} \sum_{i=1}^{N}\left(\alpha_{k}^{2}+2 \alpha_{k} \beta_{k}\left|z_{k}^{i}\right|^{\gamma}+\beta_{k}^{2}\left|z_{k}^{i}\right|^{2 \gamma}\right) \\
& +\alpha_{k} \sum_{i=1}^{N} p_{i}\left|z_{k+1}^{i}\right|+\beta_{k} \sum_{i=1}^{N} p_{i}\left|z_{k}^{i}\right|^{\gamma}\left|z_{k+1}^{i}\right|
\end{aligned}
$$

where $H=L+B$ and $Q=\left(P H+H^{T} P\right) / 2$ are used to derive the second equality. If $\sum_{i=1}^{N}\left|z_{k+1}^{i}\right| \leq 1$ holds, then we have

$$
\begin{aligned}
\dot{V}_{k} \leq & -\lambda_{\min }^{Q} \sum_{i=1}^{N}\left(\alpha_{k}^{2}+2 \alpha_{k} \beta_{k}\left|z_{k}^{i}\right|^{\gamma}+\beta_{k}^{2}\left|z_{k}^{i}\right|^{2 \gamma}\right) \\
& +\alpha_{k} \bar{p} N+\beta_{k} \bar{p} \sum_{i=1}^{N}\left|z_{k}^{i}\right|^{\gamma}
\end{aligned}
$$

Let $\alpha_{k} \triangleq \bar{p} / \lambda_{\min }^{Q}$, and for $\gamma>1, \dot{V}_{k}$ verifies

$$
\begin{aligned}
\dot{V}_{k} & \leq-\lambda_{\min }^{Q} \beta_{k} \sum_{i=1}^{N}\left(\alpha_{k}\left|z_{k}^{i}\right|^{\gamma}+\beta_{k}\left|z_{k}^{i}\right|^{2 \gamma}\right) \\
& \leq-\lambda_{\min }^{Q} \alpha_{k} \beta_{k} N^{1-\gamma}\left(\sum_{i=1}^{N}\left|z_{k}^{i}\right|\right)^{\gamma}
\end{aligned}
$$




$$
-\lambda_{\min }^{Q} \beta_{k}^{2} N^{1-2 \gamma}\left(\sum_{i=1}^{N}\left|z_{k}^{i}\right|\right)^{2 \gamma}
$$

Likewise, the following two cases are considered: Case i) For $\sum_{i=1}^{N}\left|z_{k}^{i}\right|>1$, it follows (21) that

$$
\sum_{i=1}^{N}\left|z_{k}^{i}\right| \geq\left(\frac{V_{k}}{\bar{p}\left(\alpha_{k}+\frac{\beta_{k}}{1+\gamma}\right)}\right)^{\frac{1}{1+\gamma}}
$$

Substituting (24) into (23) yields

$$
\begin{aligned}
\dot{V}_{k} \leq & -\frac{\lambda_{\min }^{Q} \alpha_{k} \beta_{k} N^{1-\gamma}}{\bar{p}^{\frac{1}{1+\gamma}}\left(\alpha_{k}+\frac{\beta_{n}}{1+\gamma}\right)^{\frac{1}{1+\gamma}}} V_{k}^{\frac{\gamma}{1+\gamma}} \\
& -\frac{\lambda_{\min }^{Q} \beta_{k}^{2} N^{1-2 \gamma}}{\bar{p}^{\frac{1}{1+\gamma}}\left(\alpha_{k}+\frac{\beta_{k}}{1+\gamma}\right)^{\frac{1}{1+\gamma}}} V_{k}^{\frac{2 \gamma}{1+\gamma}}
\end{aligned}
$$

Case ii) For $\sum_{i=1}^{N}\left|z_{k}^{i}\right| \leq 1$, from (21) we have

$$
\sum_{i=1}^{N}\left|z_{k}^{i}\right| \geq\left(\frac{V_{k}}{\bar{p}\left(\alpha_{k}+\frac{\beta_{k}}{1+\gamma}\right)}\right)
$$

Substituting (26) into (23) obtains

$$
\dot{V}_{k} \leq-\frac{\lambda_{\min }^{Q} \alpha_{k} \beta_{k} N^{1-\gamma}}{\bar{p}\left(\alpha_{k}+\frac{\beta_{k}}{1+\gamma}\right)} V_{k}^{\gamma}-\frac{\lambda_{\min }^{Q} \beta_{k}^{2} N^{1-2 \gamma}}{\bar{p}\left(\alpha_{k}+\frac{\beta_{k}}{1+\gamma}\right)} V_{k}^{2 \gamma}
$$

For the case $\gamma<1$, inductions can be carried out in a similar way. To this end, the main results are summarized in the following two theorems.

Theorem 2: If Assumptions 1 and 3 hold and the observer gains in (3) satisfy $\gamma>1$ and

$$
\begin{aligned}
& \beta_{k}>0, \quad \forall k=1,2, \ldots, n, \\
& \alpha_{k}=\frac{\bar{p}}{\lambda_{\min }^{Q}}, \quad \forall k=1,2, \ldots, n-1, \quad \alpha_{n}=\frac{b_{n} \bar{u}_{0} \bar{p}}{\lambda_{\min }^{Q}},
\end{aligned}
$$

i) the set

$$
\mathcal{S}=\mathcal{S}_{1} \cup \mathcal{S}_{2} \ldots \cup \mathcal{S}_{n} \quad \text { with } \quad \mathcal{S}_{k}=\left\{z_{k}\left|\sum_{i=1}^{N}\right| z_{k}^{i} \mid \leq 1\right\}
$$

is fixed-time attractive with the settling time bounded by

$$
T_{o}=\sum_{k=1}^{n} t_{k}
$$

where

$$
\begin{aligned}
t_{k} & =\frac{1+\gamma}{\alpha_{k} N^{1-\gamma} c_{k}}+\frac{1+\gamma}{\beta_{k} N^{1-2 \gamma} c_{k}(\gamma-1)}, \\
c_{k} & =\frac{\lambda_{\min }^{Q} \beta_{k}}{\bar{p}^{\frac{1}{1+\gamma}}\left(\alpha_{k}+\frac{\beta_{k}}{1+\gamma}\right)^{\frac{1}{1+\gamma}}}, \quad k=1,2, \ldots, n .
\end{aligned}
$$

ii) the origin of the observation disagreement dynamics (8) is globally asymptotically stable.

Proof: The proof proceeds in three steps. The basic idea is to prove the result in a recursive fashion, and thus we have to show first that no finite-time escape occurs, i.e., forward completeness of (8). Then, we show that the set $\mathcal{S}$ is fixed-time attractive. Finally, the proof is finished by showing that the origin of (8) is asymptotically stable.

(i) To show the system (8) is forward complete ${ }^{1}$, we consider the following positive definite function

$$
V=\sum_{k=1}^{n} V_{k}=\sum_{k=1}^{n} \sum_{i=1}^{N} p_{i}\left(\alpha_{k}\left|z_{k}^{i}\right|+\frac{\beta_{k}}{1+\gamma}\left|z_{k}^{i}\right|^{1+\gamma}\right) .
$$

${ }^{1}$ System (1) is called forward complete if for every initial condition $x_{0}$, the corresponding solution $x\left(t, x_{0}\right)$ is defined for all $t \geq 0$.
Using (16) and (22), the time derivative of $V$ satisfies

$$
\begin{gathered}
\dot{V} \leq \sum_{k=2}^{n}\left(\alpha_{k-1} \sum_{i=1}^{N} p_{i}\left|z_{k}^{i}\right|+\beta_{k-1} \sum_{i=1}^{N} p_{i}\left|z_{k-1}^{i}\right|^{\gamma}\left|z_{k}^{i}\right|\right) \\
\leq \sum_{k=2}^{n}\left(\alpha_{k-1} \sum_{i=1}^{N} p_{i}\left|z_{k}^{i}\right|+\frac{\beta_{k-1} \gamma}{1+\gamma} \sum_{i=1}^{N} p_{i}\left|z_{k-1}^{i}\right|^{1+\gamma}\right. \\
\left.\quad+\frac{\beta_{k-1}}{1+\gamma} \sum_{i=1}^{N} p_{i}\left|z_{k}^{i}\right|^{1+\gamma}\right) \\
\leq \sum_{k=1}^{n}\left(\alpha_{k} \sum_{i=1}^{N} p_{i}\left|z_{k}^{i}\right|+\beta_{k} \sum_{i=1}^{N} p_{i}\left|z_{k}^{i}\right|^{1+\gamma}\right) \\
\leq c V
\end{gathered}
$$

where Lemma 5 is invoked to derive the second inequality with $\zeta=$ 1 , and $c$ denote some constant. Thus, no finite-time escape ${ }^{2}$ exists, i.e., the system (8) is forward complete.

(ii) The fixed-time attractivity of set $\mathcal{S}$ will be proved recursively. Consider the Lyapunov function (15) for dynamics of $z_{n}$. Note from $\gamma>1$ that $0<\frac{\gamma}{1+\gamma}<1<\frac{2 \gamma}{1+\gamma}$. By Lemma 2, it has been shown from (18) that set $\mathcal{S}_{n}=\left\{z_{n}\left|\sum_{i=1}^{N}\right| z_{n}^{i} \mid \leq 1\right\}$ is attained within finite time bounded by $t_{n}$ defined in (31) and the trajectory $z_{n}$ will remain in $\mathcal{S}_{n}$ for all $t>t_{n}$. For $\sum_{i=1}^{N}\left|z_{n}^{i}\right| \leq 1$, we have shown in Step $k$ that (25) holds for $k=n-1$, which, by Lemma 2, implies that $\mathcal{S}_{n-1}=\left\{z_{n-1}\left|\sum_{i=1}^{N}\right| z_{n-1}^{i} \mid \leq 1\right\}$ is fixed-time attractive and $z_{n-1}$ will remain in $\mathcal{S}_{n-1}$ for all $t>t_{n-1}+t_{n}$. Recursively, we can show that $z_{1}$ attains set $\mathcal{S}_{1}$ and stay in it for all $t>\sum_{k=1}^{n} t_{k}$. Thus, set $\mathcal{S}$ is fixed-time attractive with settling time bounded by $T_{o}$ as shown in (30).

(iii) For all $t>T_{o}$, all trajectories $z_{k}, k=1,2, \ldots, n$ remain in set $\mathcal{S}$. We have shown in Case ii) of Step 1 that dynamics of $z_{n}$ is asymptotically stable at the origin due to $\gamma>1$. Similarly, it follows from (27) in Step $k$ that the origin of dynamics $z_{k}$ is asymptotically stable. Recalling $z_{k}=\left[z_{k}^{1}, z_{k}^{2}, \ldots, z_{k}^{N}\right]^{T}$ with its element defined in (7), we have $z_{k}=(L+B) \tilde{\chi}_{k}=H \tilde{\chi}_{k}$ for all $k=1,2, \ldots, n$. By invoking Lemma 7, $H=L+B$ is of full rank if Assumption 3 holds. Hence, the observation errors $\tilde{\chi}_{k}$ approaches zero asymptotically if and only if $z_{k}$ is asymptotically stable at the origin. This completes the proof.

Theorem 3: If Assumptions 1 and 3 hold and the observer gains in (3) satisfy (28)-(29) and $0.5<\gamma<1$, then, the origin of the observation disagreement dynamics (8) is globally finite-time stable with the settling time bounded by

$$
T_{o}=\sum_{k=1}^{n}\left(\tau_{k}+\bar{\tau}_{k}\right)
$$

with

$$
\begin{aligned}
& \tau_{k}=\min \left\{\frac{1+\gamma}{\alpha_{k} c_{k}}\left(V_{k}(0)\right)^{\frac{1}{1+\gamma}},\right. \\
& \bar{\tau}_{k}\left.=\frac{1+\gamma}{\beta_{k} c_{k} N^{1-2 \gamma}(1-\gamma)}\left(V_{k}(0)\right)^{\frac{1-\gamma}{1+\gamma}}\right\} \\
& \frac{1}{\alpha^{1-\gamma} \bar{c}_{k}(1-\gamma)}+\frac{1}{\beta_{k} N^{1-2 \gamma} \bar{c}_{k}(2 \gamma-1)}
\end{aligned}
$$

where $V_{k}(0), k=1,2, \ldots, n$, is the initial value of $V_{k}\left(z_{k}(t)\right)$ at $t=0$

$$
c_{k}=\frac{\lambda_{\min }^{Q} \beta_{k}}{\bar{p}^{\frac{1}{1+\gamma}}\left(\alpha_{k}+\frac{\beta_{k}}{1+\gamma}\right)^{\frac{1}{1+\gamma}}}, \quad \text { and } \quad \bar{c}_{k}=\frac{\lambda_{\min }^{Q} \beta_{k}}{\bar{p}\left(\alpha_{k}+\frac{\beta_{k}}{1+\gamma}\right)} .
$$

${ }^{2}$ The phrase finite-time escape is used to describe the phenomenon that a trajectory escapes to infinity at a finite time. 
Proof: Following the same line as the proof of Theorem 2, the forward completeness of (8) can be ensured, and due to $1 / 2<\gamma<1$ and $2 \gamma>1$, Lemmas 3 and 4 are invoked for (18) and (25) to derive that

$$
\dot{V}_{k} \leq-\lambda_{\min }^{Q} \alpha_{k} \beta_{k}\left(\sum_{i=1}^{N}\left|z_{k}^{i}\right|\right)^{\gamma}-\lambda_{\min }^{Q} \beta_{k}^{2} N^{1-2 \gamma}\left(\sum_{i=1}^{N}\left|z_{k}^{i}\right|\right)^{2 \gamma}
$$

For $\sum_{i=1}^{N}\left|z_{k}^{i}\right|>1, k=1,2, \ldots, n$, by substituting (24) into (37) and noting that $0<\frac{\gamma}{1+\gamma}<\frac{2 \gamma}{1+\gamma}<1$, we obtain

$$
\dot{V}_{k} \leq-\alpha_{k} c_{k} V_{k}^{\frac{\gamma}{1+\gamma}} \quad \text { or } \quad \dot{V}_{k} \leq-\beta_{k} c_{k} N^{1-2 \gamma} V_{k}^{\frac{2 \gamma}{1+\gamma}} .
$$

Applying Lemma 1 for (38), one obtains that the set $\mathcal{S}_{k}$ is finite-time attractive and the settling time can be estimated based on either one in (38). To be less conservative, the smaller estimate is employed, as shown in (35). In a recursive manner, it can be concluded that the trajectory $z_{k}$ will enter and remain in $\mathcal{S}_{k}$ sequentially for all $t>\sum_{l=1}^{k} \tau_{l}$. Once the trajectory enters $\mathcal{S}_{k}$, we have by substituting (26) into (37) that $\dot{V}_{k} \leq-\alpha_{k} \bar{c}_{k} V_{k}^{\gamma}-\beta_{k} \bar{c}_{k} N^{1-2 \gamma} V_{k}^{2 \gamma}$. Hence, the origin of (8) is fixed-time stable by noting $0.5<\gamma<1<2 \gamma$ and Lemma 2. In each $\mathcal{S}_{k}$, the respective settling time can be calculated as (36). Hence, the time for the whole response transition is bounded by $T_{o}$ shown in (34). Finally, we can conclude from the fact $z_{k}=H \tilde{\chi}_{k}$ and Lemma 7 that the observation errors $\tilde{\chi}_{k}, k=1,2, \ldots, n$, are finite-time stable at the origin with the settling time bounded by (34). This completes the proof.

Remark 6: Note that the convergence time estimate (34) given in Theorem 3 depends on the initial values $z_{k}^{i}(0)=$ $\sum_{j=1}^{N} a_{i j}\left(\hat{x}_{0, k}^{j}(0)-\hat{x}_{0, k}^{i}(0)\right)-b_{i}\left(\hat{x}_{0, k}^{i}(0)-x_{0, k}(0)\right)$, which makes the estimation inhibitive if the initial condition of the leader state $x_{0, k}(0)$ is unknown. Although the set fixed-time stability can be achieved by selecting $\gamma>1$, the origin is only global asymptotically stable, as shown by Theorem 2 .

Remark 7: Note also that Assumption 1 is required in the consensus observer design via condition (29). To be specific, observer gain $\alpha_{n}$ is closely related to the upper bound $\bar{u}_{0}$, which implies from (3) that a more exact estimate of $\bar{u}_{0}$ leads to a less chattering in the response of state $x_{n}$. However, it can be seen from (30) and (34) that a larger $\bar{u}_{0}$ results in a smaller estimate of the settling time and therefore may improve the convergence rate.

Remark 8: Similar to the discussion in Remark 5, it is clear that condition (29) requires the knowledge of eigenvalue information of $H$ associated with the communication graph to guarantee the fixed-time stability. After the design, the observers will work in a distributed manner. In particular, for directed graphs, this is a common feature in the existing works, e.g. [30], [31], that some global information is required for stability analysis, even if the asymptotic behavior is pursued by letting $\gamma=1$ in (3). A possible way to overcome this limitation is to use a conservative estimated version of the eigenvalue of $H$ such that (29) holds. Besides, we also note that to achieve fully distributed design, one may use the adaptive technique as shown in [32] where an adaptive consensus protocol with time-varying coupling weights is proposed to eliminate the dependence on global information. However, to achieve the finite- or fixed-time stability, it is still an open problem to design fully distributed adaptive consensus observer under directed graphs.

Remark 9: It is worth mentioning that the fixed-time stability at the origin can be achieved by introducing an additional term with a fractional exponent if $\gamma>1$ into observer (3) when the interaction topology is directed. The proof given in [21] can be extended and applied for the high-order integrator multi-agent system. However, one more term added into (3) will introduce more parameters to be tuned and the settling time estimation will be more conservative.

\section{Simulation}

The multi-agent system consists of one leader labeled by 0 and five $(N=5)$ followers labeled by 1 to 5 . The dynamics of the leader is described by

$$
\dot{x}_{0,1}(t)=x_{0,2}(t), \quad \dot{x}_{0,2}(t)=x_{0,3}(t), \quad \dot{x}_{0,3}(t)=u_{0}(t),
$$

where $x_{0,1}, x_{0,2}$ and $x_{0,3}$, denote the position, the velocity and the acceleration, respectively (i.e., $n=3$ ), $u_{0}$ is the input of the leader. In simulation, the input of the leader is selected as $u_{0}=-\sin (0.5 t)$ which is bounded by $\bar{u}_{0}=1$. The initial values of the leader are fixed as $x_{0}=[-8,0,2]^{T}$. The simulation is carried out using Euler method with a fixed integration step equal to $10^{-4}$ [33]. In order to validate the theoretical findings, two scenarios for different initial values of the consensus observers are considered: (i) $\hat{x}_{0}^{1}=[0,0,0]$, $\hat{x}_{0}^{2}=[0,0,0], \hat{x}_{0}^{3}=[0,0,0], \hat{x}_{0}^{4}=[0,0,0], \hat{x}_{0}^{5}=[0,0,0]$ and (ii) $\hat{x}_{0}^{1}=[-10,0,0], \hat{x}_{0}^{2}=[-5,0,0], \hat{x}_{0}^{3}=[2,0,0], \hat{x}_{0}^{4}=[7,0,0]$, $\hat{x}_{0}^{5}=[20,0,0]$; and simulation studies have been performed under two different interconnection graphs.

\section{A. Results for Undirected Interaction Topology}

The information flow among followers is undirected and connected, as shown in Fig. 1. Then, we have $\lambda_{\max }^{H}=4.9032$ and $\lambda_{\min }^{H}=$ 0.2907 . Let $\varepsilon=0.2$ and $\gamma=2$. The observer parameters $\alpha_{1}=$ $\alpha_{2}=0.58, \alpha_{3}=1.58, \beta_{1}=\beta_{2}=\beta_{3}=0.59$ and upper bound of the settling time $T_{o}=15 \mathrm{sec}$ can be computed according to Theorem 1. The simulation results are displayed in Fig. 2, from which we can see that all estimated positions produced by the distributed observers converge to the leader's position within about $7 \mathrm{sec}$ although different initial conditions are implemented.

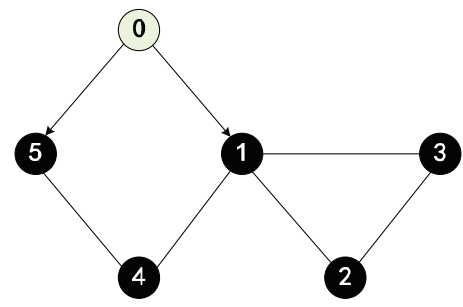

Fig. 1. Undirected Information flow

\section{B. Results for Directed Interaction Topology}

The information flow among followers is directed and contains a spanning tree with the leader as the root node, as shown in Fig. 3. By Lemma 7, we have $p=[4.5,6.5,5.5,1.5,0.5]^{T}$. Then, we have that $\bar{p}=6.5, \lambda_{\min }^{Q}=0.8325$, and thus $\alpha_{1}=\alpha_{2}=\alpha_{3}=7.8$ can be computed by Theorem 2. Let $\beta_{1}=\beta_{2}=\beta_{3}=0.2$ and $\gamma=2$. From the simulation results shown in Fig. 4, we can see that the distributed observers under directed information flow achieves fast convergence to the leader state. Furthermore, under different initial conditions, the settling times are almost the same, which demonstrate the fixed-time attractivity property.

To validate the statement of Theorem 3, we set $\gamma=0.6$ and the other parameters are not changed. It is observed from Fig. 5 that larger initial disagreement leads to longer settling time, which demonstrates the finite-time stability of the proposed observer for $\gamma<1$. 


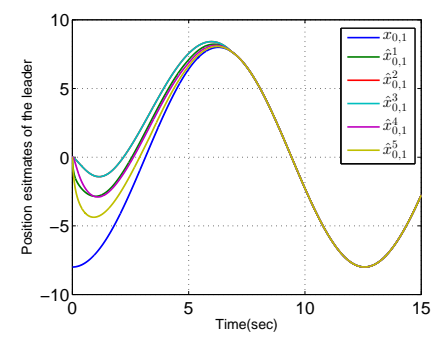

(a) Scenario (i)

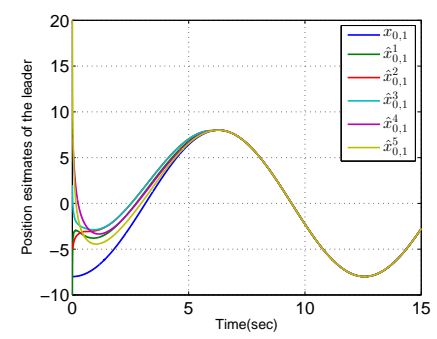

(b) Scenario (ii)

Fig. 2. Profiles of the estimated positions subject to undirected Graph: $\gamma=2$

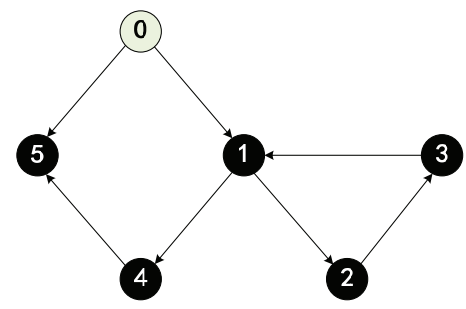

Fig. 3. Directed Information flow

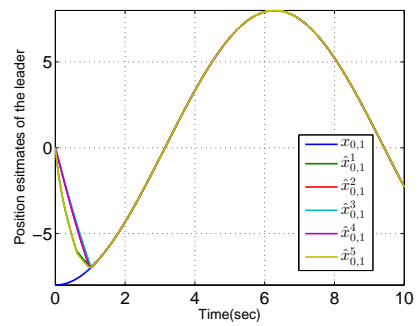

(a) Scenario (i)

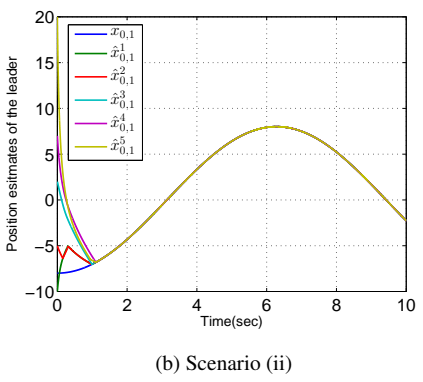

Fig. 4. Profiles of the estimated positions subject to directed graph: $\gamma=2$

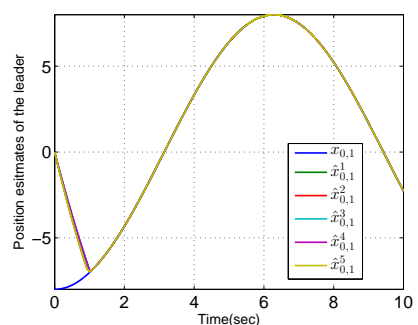

(a) Scenario (i)

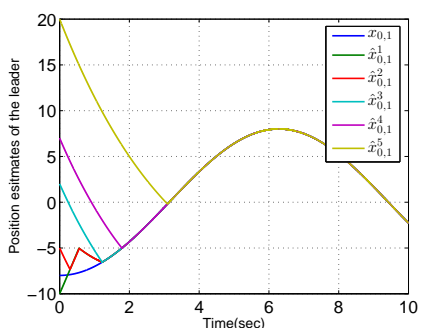

(b) Scenario (ii)

Fig. 5. Profiles of the estimated positions subject to directed graph: $\gamma=0.6$

Finally, we want to show how the performance changes with respect to the different upper bounds on the leader input. Thus, consider a conservative upper bound estimate $\bar{u}_{0}=10$ for the comparison and another set of initial conditions for the convenience: (iii) $\hat{x}_{0}^{1}=$ $[-10,0,12], \hat{x}_{0}^{2}=[-5,0,0], \hat{x}_{0}^{3}=[2,0,-5], \hat{x}_{0}^{4}=[7,0,0], \hat{x}_{0}^{5}=$ $[20,0,-9]$. By (29), a new $\alpha_{3}=78$ can be computed accordingly. Without tuning the other gains, the simulation results are shown in Figs. 6(b) and 7(b). By comparing with Figs. 6(a) and 7(a) under the exact knowledge of the upper bound, we can see that the larger $\bar{u}_{0}$ results in a faster convergence rate and a bigger chattering in the acceleration responses. However, the position responses are more insensitive to the upper bound $\bar{u}_{0}$ and smoother due to the high order structure. By a very closer inspection of Fig. 6 , the convergence rate of the estimated position is slightly faster with the bigger $\bar{u}_{0}$.

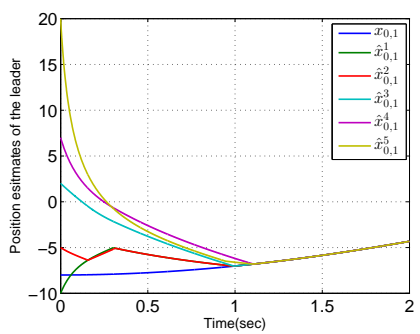

(a) $\bar{u}_{0}=1$ and $\alpha_{3}=7.8$

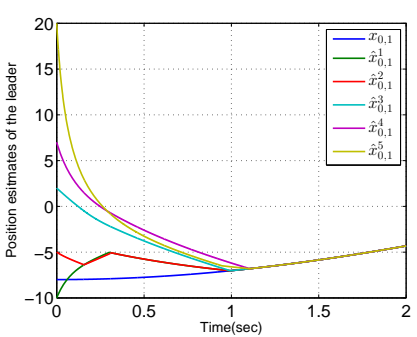

(b) $\bar{u}_{0}=10$ and $\alpha_{3}=78$

Fig. 6. Profiles of the estimated positions subject to directed graph: Scenario (iii) and $\gamma=2$

In summary, the proposed distributed consensus observer has interesting features: the stability type is closely related to the interaction 


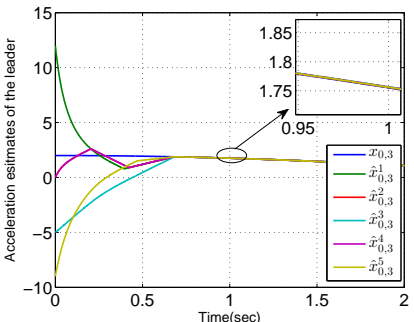

(a) $\bar{u}_{0}=1$ and $\alpha_{3}=7.8$

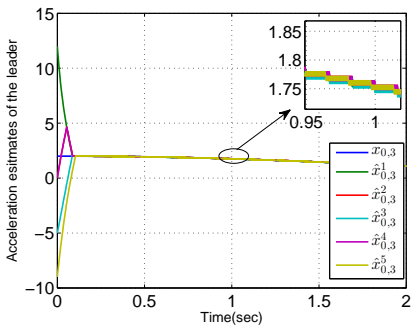

(b) $\bar{u}_{0}=10$ and $\alpha_{3}=78$

Fig. 7. Profiles of the estimated accelerations subject to directed graph: Scenario (iii) and $\gamma=2$

topology and the exponent we set. From the simulation results, we also found that the settling time estimate derived for directed case is much more conservative than the one for undirected case.

\section{CONCLUSIONS}

This paper studies a new distributed consensus observer, which is implemented in a group of agents in leader-follower setting to estimate the leader state. The stability analysis is carried out within the Lyapunov framework and shows that different stability features are achieved by properly selecting the exponent in the observer design. Future work may include the exploration of less conservative estimation for the settling time and the application of the proposed observer in consensus design.

\section{REFERENCES}

[1] R. Olfati-Saber and R. M. Murray, "Consensus problems in networks of agents with switching topology and time delays," IEEE Transaction on Automatic Control, vol. 49, no. 9, pp. 1520-1533, 2004.

[2] W. Ren and R. Beard, "Consensus seeking in multiagent systems under dynamically changing interaction topologies," IEEE Transaction on Automatic Control, vol. 50, no. 5, pp. 655-661, 2005.

[3] Z. Ding, "Adaptive consensus output regulation of a class of nonlinear systems with unknown high-frequency gain," Automatica, vol. 51, pp. 348-35, 2015

[4] A. Abdessameud and A. Tayebi, "Formation control of vtol unmanned aerial vehicles with communication delays," Automatca, vol. 47, no. 11, pp. 2383-2394, 2011

[5] A.-S.-M. Isira, Z. Zuo, and Z. Ding, "Leader-follower consensus control of lipschitz nonlinear systems by output feedback," International Journal of Systems Science, vol. 47, no. 16, pp. 3772-3781, 2016.

[6] Y. Cao, L. Zhang, C. Li, and M.-Z.-Q. Chen, "Observer-based consensus tracking of nonlinear agents in hybrid varying directed topology," IEEE Transactions Cybernetics, vol. 8, no. 47, pp. 2212-2222, 2017.

[7] X. Liu, D.-W.-C. Ho, J. Cao, and W. Xu, "Discontinuous observers design for finite-time consensus of multiagent systems with externa disturbances," IEEE Transactions on Neural Network and Learning Systems, vol. 28, no. 11, pp. 2826-2830, 2017.

[8] A. Polyakov, "Nonlinear feedback design for fixed-time stabilization of linear control systems," IEEE Transactions on Automatic Control, vol. 57, no. 8, pp. 2106-2110, 2012.

[9] S. Parsegov, A. Polyakov, and P. Shcherbakov, "Fixed-time consensus algorithm for multi-agent systems with integrator dynamics," in 4th IFAC Workshop on Distributed Estimation and Control in Networked Systems, Koblenz, Germany, Sept. 2013, pp. 110-115.
[10] B. Tian, Z. Zuo, X. Yan, and H. Wang, "A fixed-time output feedback control scheme for double integrator systems," Automatica, pp. 17-24, 2017.

[11] Z. Zuo, Q.-L. Han, B. Ning, X. Ge, and X.-M. Zhang, "Non-singular fixed-time consensus tracking for second-order multi-agent networks," IEEE Transactions on Industrial Informatics, vol. 14, no. 6, pp. 23222334, 2018

[12] Z. Zuo and L. Tie, "A new class of finite-time nonlinear consensus protocols for multi-agent systems," International Journal of Control, vol. 87, no. 2, pp. 363-370, 2014.

[13] Z. Zuo, "Non-singular fixed-time consensus tracking for second-order multi-agent networks," Automatica, vol. 54, no. 4, pp. 305-309, 2015.

[14] M. Defoort, A. Polyakov, G. Demesure, M. Djemai, and K. Veluvolu, "Leader-follower fixed-time consensus for multi-agent systems with unknown non-linear inherent dynamics," IET Control Theory \& Applications, vol. 9, no. 14, pp. 2165-2170, 2015.

[15] M. Defoort, G. Demesure, Z. Zuo, A. Polyakov, and M. Djemai, "Fixedtime stabilisation and consensus of non-holonomic systems," IET Control Theory \& Applications, vol. 10, no. 18, pp. 2497-2505, 2016.

[16] B. Tian, H. Lu, Z. Zuo, and W. Yang, "Fixed-time leader-follower output feedback consensus for second-order multiagent systems," IEEE transactions on cybernetics, no. 4, pp. 1545-1550, 2019.

[17] A. Levant, "Higher-order sliding modes, differentiation and output feedback control," International Journal of Control, vol. 76, no. 9/10, pp. 924-641, 2003.

[18] M. Angulo, J. Moreno, and L. Fridman, "Robust exact uniformly convergent arbitrary order differentiator," Automatica, vol. 49, no. 8 pp. 2489-2495, 2013.

[19] H.-G. Marina, M. Cao, and B. Jayawardhana, "Controlling rigid formations of mobile agents under inconsistent measurements," IEEE Transactions on Robotics, vol. 31, no. 1, pp. 31-39, 2015.

[20] J. Fu and J. Wang, "Fixed-time coordinated tracking for second-order multi-agent systems with bounded input uncertainties," Systems \& Control Letters, vol. 93, pp. 1-12, 2016.

[21] — " "Finite-time consensus for mulit-agent systems with globally bounded convergence time under directed communiction graphs," International Journal of Control, vol. 90, no. 9, pp. 1807-1817, 2017.

[22] Z. Zuo, B. Tian, M. Defoort, and Z. Ding, "Fixed-time consensus tracking for multi-agent systems with high-order integrator dynamics," IEEE Transactons on Automatic Control, vol. 63, no. 2, pp. 563-570, 2018.

[23] S. Khoo, L. Xie, and Z. Man, "Robust finite-time consensus tracking algorithm for multirobot systems," IEEE/ASME Transactions on Mechatronics, vol. 14, no. 2, pp. 219-228, 2009.

[24] S. Khoo, L. Xie, S. Zhao, and Z. Man, "Multi-surface sliding control for fast finite-time leader-follower consensus with high order siso uncertain nonlinear agents," International Journal of Robust and Nonlinear Control, vol. 24, no. 6, pp. 2388-2404, 2014.

[25] H. Zhang, Z. Li, Z. Qu, and F. Lewis, "On constructing lyapunov functions for multi-agent systems," Automatica, vol. 58, pp. 39-42, 2016.

[26] A. Filippov, Differential equations with discontinuous right-hand side. Kluwer Academic, Dordrecht, 1988.

[27] S. Bhat and D. Bernstein, "Finite time stability of continuous autonomous systems," SIAM Journal of Control Optimization, vol. 38, no. 3, pp. 751-766, 2000.

[28] C. Qian and W. Lin, "A continuous feedback approach to global strong stabilization of nonlinear systems," IEEE Transactions Automatic Control, vol. 46, no. 7, pp. 1061-1079, 2001.

[29] H. Zhang and F. Lewis, "Adaptive cooperative tracking control of higherorder nonlinear systems with unknown dynamics," Automatica, vol. 48, no. 7, pp. 1432-1439, 2012.

[30] J. Seo, H. Shim, and J. Back, "Consensus of high-order linear systems using dynamic output feedback compensator: Low gain approach," Automatica, vol. 45, no. 11, pp. 2659-2664, 2009.

[31] H. Zhang, F. Lewis, and A. Das, "Optimal design for synchronization of cooperative systems: State feedback, observer, output feedback," IEEE Transactions on Automatic Control, vol. 56, no. 8, pp. 1948-1952, 2011.

[32] Z. Li, G. Wen, Z. Duan, and W. Ren, "Designing fully distributed consensus protocols for linear multi-agent systems with directed graphs," IEEE Transactions on Automatic Control, vol. 60, no. 4, pp. 1152-1157, 2015.

[33] A. Levant, "On fixed and finte time stability in sliding mode control," in 52nd IEEE Conference on Decision and Control, Florence, Italy, Dec. 2013, pp. 4260-4265. 